\title{
Large Scale In Vivo Recording of Sensory Neuron Activity with GCaMP6
}

\author{
(Dim I. Chisholm, Nikita Khovanov, Douglas M. Lopes, Federica La Russa, and ${ }^{-}$Stephen B. \\ McMahon
}

DOI:http://dx.doi.org/10.1523/ENEURO.0417-17.2018

Neurorestoration Group, Wolfson Centre for Age-Related Diseases, King's College London, London SE1 1UL, United Kingdom

\begin{abstract}
Greater emphasis on the study of intact cellular networks in their physiological environment has led to rapid advances in intravital imaging of the central nervous system (CNS), while the peripheral system remains largely unexplored. To assess large networks of sensory neurons, we selectively label primary afferents with GCaMP6s in male and female $\mathrm{C} 57 \mathrm{bl} / 6$ mice and visualize their functional responses to peripheral stimulation in vivo. We show that we are able to monitor the activity of hundreds of sensory neurons simultaneously, with sufficient sensitivity to detect, in most cases, single action potentials with a typical rise time of around $200 \mathrm{~ms}$, and an exponential decay with a time constant of approximately $700 \mathrm{~ms}$. With this technique we are able to characterize the responses of large populations of sensory neurons to innocuous and noxious mechanical and thermal stimuli under normal and inflammatory conditions. We demonstrate that the majority of primary afferents are polymodal with between $50-80 \%$ of thermally sensitive DRG neurons responding also to noxious mechanical stimulation. We also specifically assess the small population of peripheral cold neurons and demonstrate significant sensitization to cooling after a model of sterile and persistent inflammation, with significantly increased sensitivity already at decreases of $5^{\circ} \mathrm{C}$ when compared to uninflamed responses. This not only reveals interesting new insights into the (patho)physiology of the peripheral nervous system but also demonstrates the sensitivity of this imaging technique to physiological changes in primary afferents.
\end{abstract}

Key words: dorsal root ganglia; genetically encoded calcium indicators; in vivo imaging; nociception; pain; primary afferents

\section{Significance Statement}

Most of our functional understanding of the peripheral nervous system has come from single unit recordings. However, the acquisition of such data are labor intensive and usually "low yield." Moreover, some questions are best addressed by studying populations of neurons. To this end we report on a system that monitors activity in hundreds of single sensory neurons simultaneously, with sufficient sensitivity to detect in most cases single action potentials. We use this technique to characterize response properties to natural and electrical stimuli and to demonstrate polymodality in the majority of neurons as well as their sensitization under inflammatory conditions. We therefore believe this approach will be very useful for the study of the somatosensory system in general, and pain in particular.

\section{Introduction}

Primary somatosensory neurons and their cell bodies located in the dorsal root ganglion (DRG) are functionally, anatomically, physiologically and genetically diverse (Kandel

Received December 5, 2017; accepted January 30, 2018; First published February 26, 2018.

The authors declare no competing financial interests. et al., 2013; Usoskin et al., 2015). These neurons are critical for a large number of distinct sensations including, but not limited to, touch, pain, itch, proprioception and temperature. Our knowledge regarding the encoding properties of these 
neurons is largely derived from single unit recording studies of individual afferent fibers, work that is hampered by the low throughput of such data acquisition.

The advent of genetically encoded calcium indicators has opened up the possibility for large scale optical assessment of the functional and morphologic characteristics of entire neuronal networks with good spatial and temporal sensitivity. These techniques have been applied to a variety of CNS structures, including sensory, motor, and visual cortex and spinal cord (Stosiek et al., 2003; Dombeck et al., 2007; Flusberg et al., 2008; Tian et al., 2009; Johannssen and Helmchen, 2010; Ghosh et al., 2011; Chen et al., 2012, 2013; Zariwala et al., 2012; Sun et al., 2013; Dana et al., 2014; Sekiguchi et al., 2016) and, very recently, some peripheral networks (Barretto et al., 2015; Williams et al., 2016; Wu et al., 2015). In recent months, a few groups have also described the application of in vivo imaging to the DRG (Emery et al., 2016; Kim et al., 2016; Smith-Edwards et al., 2016), but due to the novelty of the technique the peripheral nervous system still remains relatively unexplored.

Indeed, it is evident from this small string of papers that the application of in vivo imaging to the peripheral nervous system is still an evolving field. For example, an initially slower version of the calcium indicator GCaMP, as well as very slow image acquisition speeds, may have hampered a more detailed analysis of the pathophysiology of the peripheral nervous system (Kim et al., 2016; Smith-Edwards et al., 2016), while small numbers of sampled neurons could reduce the benefits inherent to this approach (Emery et al., 2016; Smith-Edwards et al., 2016). For example, one such recent publication suggested that the vast majority of primary afferents exhibit modality specificity (Emery et al., 2016). This is in stark contrast to the frequently observed (and widely reported) nociceptive polymodality seen using microneurography and electrophysiology, in addition to multiple lines of in vitro evidence (as reviewed in chapter 1 of McMahon et al., 2013). Here, we reexamine this claim using large scale in vivo GCaMP imaging, and confirm traditional views of widespread primary afferent polymodality.

This work was supported by the Wellcome Trust 102645/Z/13/Z.

Acknowledgment: The Genetically-Encoded Neuronal Indicator and Effector (GENIE) Project and the Janelia Research Campus of the Howard Hughes Medical Institute has generously allowed this material to be distributed with the understanding that requesting investigators need to acknowledge the GENIE Program and the Janelia Research Campus in any publication in which the material was used, specifically Vivek Jayaraman, Ph.D., Douglas S. Kim, Ph.D., Loren L. Looger, Ph.D., and Karel Svoboda, Ph.D., from the GENIE Project, Janelia Research Campus, Howard Hughes Medical Institute. We thank Professor Peter McNaughton and Tamara Buijs for the use of Snap25-2AGCaMP6s-D mice, Tatum Cummins and Dr. Franziska Denk for valuable comments on the MS, and Dr. Tommaso Tufarelli and Dr. Sarah Chisholm for expert statistical advice.

Correspondence should be addressed to Neurorestoration Group, Wolfson Wing, Hodgkin Building, King's College London, Guy's Campus, London Bridge, London SE1 1UL, United Kingdom, E-mail: kim.chisholm@kcl.ac.uk.

DOI:http://dx.doi.org/10.1523/ENEURO.0417-17.2018

Copyright (C) 2018 Chisholm et al.

This is an open-access article distributed under the terms of the Creative Commons Attribution 4.0 International license, which permits unrestricted use, distribution and reproduction in any medium provided that the original work is properly attributed.
We present an enhanced method which provides for the first time the added benefits of being able to sample from hundreds of neurons simultaneously, at acquisition frequencies above $1 \mathrm{~Hz}$, using very low light levels with no detectable photo-bleaching and an increased resistance to sample movement. These features make this technique useful to the study of large populations of heterogeneous neurons responding variably across time. With this technique, we are therefore able to sample the diverse array of DRG neurons in response to acute and persistent pain states in vivo.

\section{Materials and Methods}

For all experiments, aside from polymodality experiments, we used adult C57BL/6J mice (Envigo) and expressed GCaMP6s in sensory neurons via intrathecal AAV9. For comparison, we also studied responses in Cre-dependent C57BL/6 GCaMP6s mice (The Jackson Laboratory, stock no: 028866) crossed with AdvillinCreERT2 (courtesy of John Wood), and also in Snap252A-GCaMP6s-D (The Jackson Laboratory, stock no: 025111) in experiments of polymodality only (Fig. 4C). The animals weighed $20-35 \mathrm{~g}$ at the time of experimentation. Both male and female mice were housed on a 12/12 $h$ light/dark cycle with a maximum of 8 mice per cage, with food and water available ad libitum. All experiments were performed in accordance with the United Kingdom Home Office Animals (Scientific Procedures) Act (1986).

\section{Intrathecal administration of AAV9-GCaMP6s}

C57BL/6J mice were anesthetized with isoflurane ( $2 \%$ in oxygen), and Carprieve (0.025 mg; Norbrook Laboratories) was administered subcutaneously for postoperative pain management. Mice were maintained at around $37^{\circ} \mathrm{C}$ using a homeothermic heating mat. An incision was made in the skin over the lumbar region and muscle was removed to expose the intervertebral membrane between T12 and T13 vertebrae. The region surrounding the lumbar enlargement provides easier access to the intervertebral area and ensures minimal damage. A small cut was made in the membrane and the underlying dura to insert a small catheter of 0.2-mm diameter (Braintree Scientific) in the caudal direction, through which $5 \mu$ l of AAV9.CAG. GCaMP6s.WPRE.SV40 (UPENN Vector Core, AV-1PV2833, $1.1 \times 1013 \mathrm{gc} / \mathrm{ml}$ ) was infused into the intrathecal space at $1.2 \mu \mathrm{l} / \mathrm{min}$. Due to the length of the inserted cannula, the infusion was close to the L4 DRGs. The catheter was left in place for 2 min before slow withdrawal. The incision was closed and mice were allowed to recover for between 14-37 d.

\section{Tamoxifen dosing}

Tamoxifen (T5648; Sigma-Aldrich) was dissolved in $100 \%$ ethanol to a concentration of $97.5 \mu \mathrm{g} / \mu \mathrm{l}$ and further dissolved to a working concentration of $7.8 \mu \mathrm{g} / \mu \mathrm{l}$ in wheat germ oil (Denk et al., 2015). This was placed on a shaker at room temperature for 2-3 $\mathrm{hr}$ and subsequently stored at $-20^{\circ} \mathrm{C}$. To induce GCaMP expression, mice carrying the Advillin-CreERT2 allele received $75 \mathrm{mg} / \mathrm{kg}$ tamoxifen intraperitoneally once daily for $3 \mathrm{~d}$. We waited for a 
minimum of $14 \mathrm{~d}$ to allow induction and accumulation of GCaMP6.

\section{In vivo imaging of sensory neuron activity using GCaMP responses}

For in vivo imaging, mice were anesthetized using urethane $(12.5 \% \mathrm{w} / \mathrm{v})$. An initial dose of $37.5 \mathrm{mg}$ (in a volume of $0.3 \mathrm{ml}$ ) was given IP. Further doses were given at $\sim 15$ to 20-min intervals, depending on hindlimb and corneal reflex activity, until surgical depth was achieved. The core body temperature was maintained close to $37^{\circ} \mathrm{C}$ using a homeothermic heating mat with a rectal probe (FHC). A tracheal catheter was installed and the mice breathed spontaneously. Animals were hydrated with $0.5-\mathrm{ml}$ sterile normal saline $(0.9 \%)$ administered subcutaneously. An incision was made in the skin on the back and the muscle overlying the L3, L4, and L5 DRG was removed. The bone around the L4 DRG was carefully removed in a caudalrostral direction and the underlying epineurium and dura mater over the DRG were washed and moistened with normal saline. The position of the mouse was varied between prone and lateral recumbent to orient the DRG in a more horizontal plane. The exposure was then stabilized at the neighboring vertebrae using spinal clamps (Precision Systems and Instrumentation) attached to a custommade imaging stage. The exposed cord and DRG were covered with silicone elastomer (World Precision Instruments, Ltd) to avoid drying and to maintain a physiologic environment. The mouse was then placed under the Eclipse Ni-E FN upright confocal/multiphoton microscope (Nikon) and the microscope stage was variably diagonally orientated to optimize focus on the DRG. The ambient temperature during imaging was kept at $32^{\circ} \mathrm{C}$ throughout. All images were acquired using a $10 \times$ dry objective. To obtain confocal images a 488-nm Argon ion laser line was used, while a Coherent Chameleon II laser was tuned to $920 \mathrm{~nm}$ for multiphoton imaging. GCaMP signal was collected at $500-550 \mathrm{~nm}$. Time series recordings were taken with an in-plane resolution of $512 \times 512$ pixels and a fully open pinhole for confocal image acquisition. Image acquisition varied between 1 and $16 \mathrm{~Hz}$ depending on the experimental requirements and signal strength.

\section{Activation of sensory neurons with electrical stimuli}

In some animals, the sciatic nerve on the side ipsilateral to the DRG being imaged was exposed through blunt dissection. A custom-made cuff electrode with Teflon insulated silver wire $(\varnothing 0.125 \mathrm{~mm}$; Advent Research Materials) was placed underneath and around the sciatic nerve. The preparation was isolated and stabilized using dental silicon impression compound (Heraeus Kulzer). A biphasic stimulator (World Precision Instruments) was used to deliver either individual or trains of square wave current pulses to the sciatic nerve. Pulses of $250-\mu \mathrm{s}$ duration and $250-\mu \mathrm{A}$ amplitude were used to activate myelinated (A) fibers in the sciatic nerve, and supramaximal pulses of 1-ms duration and 5-mA amplitude were used to activate all afferent fibers, both myelinated $(A)$ and unmyelinated $(C)$ axons.
Activation of sensory neurons with thermal stimuli

A Peltier device (TSAll, Medoc) with a $16 \times 16 \mathrm{~mm}$ probe was placed onto the plantar surface of the hind paw ipsilateral to the DRG being imaged. The temperature of the block was increased from a baseline temperature of $32^{\circ} \mathrm{C}$ to $50^{\circ} \mathrm{C}$ or decreased from $32^{\circ} \mathrm{C}$ to $4^{\circ} \mathrm{C}$. Temperature changes occurred as either a ramp of $1.5^{\circ} \mathrm{C} / \mathrm{s}$ to the target temperature (maintained for $10 \mathrm{~s}$ ) before returning to baseline at $4^{\circ} \mathrm{C} / \mathrm{s}$, or as increasing/decreasing incremental temperature ramps. Consecutive increments occurred as steps of $2^{\circ} \mathrm{C}$ in the case of an increase, and $5^{\circ} \mathrm{C}$ during a decrease (with a final drop of $3^{\circ} \mathrm{C}$ from $7^{\circ}$ to $4^{\circ} \mathrm{C}$ ) and a minimum of $90 \mathrm{~s}$ between incremental changes. Each individual increment involved a temperature change of $2^{\circ} \mathrm{C} / \mathrm{s}$, a holding temperature for $5 \mathrm{~s}$ and a return to baseline at $4^{\circ} \mathrm{C} / \mathrm{s}$.

\section{Activation of sensory neurons with mechanical and chemical stimuli}

Mechanical stimulation consisted of brushing or pinching of the plantar surface of the ipsilateral hind paw. To achieve the stimulation of the maximal number of receptive fields the entire plantar surface was pinched with blunt forceps across most of the surface of the paw. An effort was made to stimulate similar areas across different experiments to activate a comparable number of sensory fibers.

In some experiments, sterile normal saline and formalin ( $1.85 \%$ in saline) was injected at a volume of $20 \mu$ into the middle of the plantar surface of the ipsilateral hind paw. Responses of L4 DRG neurons were monitored for at least 30 min after injection of saline followed by an injection of formalin and a further 30 min of observation.

\section{Behavior assessment after formalin injection}

For behavioral experiments, mice were placed in a Perspex chamber on a wire mesh floor and allowed to acclimatize for at least $30 \mathrm{~min}$. After acclimatization, mice were lightly restrained and $20 \mu \mathrm{l}$ of $1.85 \%$ formalin in $0.9 \%$ saline solution was injected subcutaneously into the plantar surface of the hind paw using a 31-Gauge syringe. The animal was then put back into the chamber and its behavior was recorded for $30 \mathrm{~min}$. Pain behavior (time spent flinching, jerking or licking the injected paw) was quantified from video footage, using Etholog Software (Ottoni, 2000) in blocks of 5 min. All behavioral experiments were conducted during the light cycle of the day.

\section{UVB irradiation}

Mice were anesthetized with ketamine $(75 \mathrm{mg} / \mathrm{kg}$ in saline, Narketan, Vetoquinol) and medetomidine (0.5 $\mathrm{mg} / \mathrm{kg}$ in saline, Dormitor, Vetoquinol) and placed under a black cloth for protection. Their eyes were moistened and protected with eye gel (Viscotears, Liquid Gel, Novartis) and the plantar surface of the left paw was exposed through slits in the material and immobilised using tape. The paws of mice were exposed to $3000 \mathrm{~mJ} / \mathrm{cm}^{2}$ over $\sim 40$ min [the duration of exposure depended on the strength of the UVB lamp -TL01 fluorescent bulbs (maximum wavelength, $311 \mathrm{~nm}$ ), assessed before each irradiation using a photometer]. Control animals were anesthetized but not 
irradiated. Animals were allowed to recover for $48 \mathrm{~h}$ before in vivo imaging.

\section{Evaluation of transfection efficiency of DRGs}

Fourteen days after intrathecal injection of AAV9GCaMP6s, four mice were terminally anesthetized and transcardially perfused with PBS followed by $4 \%$ paraformaldehyde (PFA). The L4 DRGs were removed and postfixed for $2 \mathrm{~h}$ in $4 \%$ PFA before cryoprotection in 30\% sucrose (with $0.02 \%$ sodium azide) for $24 \mathrm{~h}$. They were then embedded in Optimal Cutting Temperature (TissueTek), cut into $10-\mu \mathrm{m}$ sections at $-20^{\circ} \mathrm{C}$ and mounted onto glass slides.

Once dried, DRG slices were rehydrated and blocked with $10 \%$ serum for $1 \mathrm{~h}$ before incubation with primary antibodies against $\beta$-III-tubulin (primary afferent marker; 1:1000, Promega, G712A), GFP (to visualize GCaMP6s; 1:1000, Abcam, ab13970), neurofilament 200 (NF200; large myelinated neurons; 1:160, Sigma Aldrich, N4142), calcitonin gene-related peptide (CGRP; small peptidergic neurons; 1:500, Enzo Life Sciences, CA1134), and isolectin B4 (IB4; conjugated to Alexa Fluor 647; small, nonpeptidergic fibers; 1:250, Invitrogen, I32450) overnight at room temperature. Slides were then incubated with the appropriate fluorophore-conjugated secondary antibodies (goat anti-chicken, Alexa Fluor 488, Invitrogen, A-11039, for GFP; goat anti-rabbit, Alexa Fluor 594, Invitrogen, A-11037, for CGRP and NF200; goat anti-mouse, Alexa Fluor 647, Invitrogen, A-32728, for $\beta$-III-tubulin, all used at $1: 1000$ dilution) for $2 \mathrm{~h}$ at room temperature. Slides were coverslipped using DAPI-containing media (Fluoromount-G with DAPI, eBioscience) and imaged with an LSM 710 laser-scanning confocal microscope (Zeiss).

\section{Experimental design and statistical analysis}

Drift in time-lapse recordings were corrected using NIS Elements AR 4.30.01 (Nikon, align application). Further image processing was done using Fiji/lmageJ version $1.48 \mathrm{v}$, and graphing and statistical analysis was undertaken with a combination of Microsoft Office Excel 2013, IBM SPSS Statistics 23 package and RStudio 0.99.893. All tests conducted were two-tailed, and $p<0.05$ was considered significant. Statistical tests and sample sizes are fully recorded in the appropriate figure legends.

To generate traces of calcium signals from time lapse images, regions of interest (ROIs) surrounding cell bodies were chosen using a free hand selection tool in Fiji. ROls were chosen with minimal overlap to ensure less interference from surrounding somata.

A region of background was selected and its signal subtracted from each ROI. To generate normalised data, a baseline period of fluorescence was recorded for each $\mathrm{ROI}$ and changes from this baseline fluorescence were calculated as $\Delta F / F$.

$$
\Delta F / F=\frac{F_{t}-F_{0}}{F_{0}}
$$

Where $F_{t}$ is the fluorescence at time $t$ and $F_{0}$ is the fluorescence average over a baseline period. Unless otherwise stated $\Delta F / F$ is expressed as a percentage.
The threshold for a positive response was taken as an average signal of $70 \%$ above baseline fluorescence plus 4 SD.

For tests of polymodality, a high level of stringency was used. To ensure that overlapping cell bodies were not contaminating the signal, and that low levels of response were not missed, all thermally responsive neurons were double checked visually for their response to pinch.

For histologic analysis, to assess the success of labeling with AAV9, six to eight neuronal cell bodies with the lowest fluorescence were selected and their average intensity plus 1 SEM was considered the cutoff for a positive signal against which all other neurons were assessed.

\section{Results}

\section{Intrathecal injections of AAV9 label a large and representative subset of neurons in L4 DRGs}

To assess efficiency of transfection, four mice, intrathecally injected with AAV9-GCaMP6, were studied histologically 14 d postinjection. In all animals, GCaMP labeling was detected using immunohistochemistry with a GFP antibody, and all neurons were identified with a $\beta$-III-tubulin antibody. A mean of $62 \pm 12 \%$ of $\beta$-III-tubulin antibody positive neurons were labeled with GCaMP6s following intrathecal injection (Fig. 1). We also observed that $100 \%$ of GCaMP6s labeled cells were $\beta$-III-tubulin-positive, which suggests that transduction was limited to neurons. Further, we were able to stain a representative population of large myelinated (NF200-positive, $60 \pm 10 \%$ ), small peptidergic (CGRP-positive, $60 \pm 10 \%$ ), and small nonpeptidergic (IB4-positive, $51 \pm 10 \%$ ) neurons with GCaMP, suggesting pan-neuronal tropism (Fig. 1).

\section{In vivo imaging of GCaMP6-labeled neurons: confocal versus two-photon microscopy}

We exposed and visualized the L4 DRG in vivo and were able to detect consistent labeling as early as two weeks after AAV9-GCaMP6 injection. The primary stability for imaging was provided by clamping the spinous process above and below the L4 vertebra with forceps (Fig. 2A). Rotation of the mouse and spinal cord position in the horizontal axis optimised the orientation of the DRG toward the optical plane of the objective and ensured maximal focus of DRGs during in vivo imaging.

We found that confocal microscopy with a maximally opened pinhole offered several advantages over multiphoton microscopy in our preparation. Confocal imaging provided more available signal, including from out-of-focus neurons (Fig. 2B). As a result, more information could be extracted from a larger number of somata (Fig. $2 B, C$ ). In addition, the greater optical slice thickness reduced the negative effects of motion and provided more stable traces of fluorescence over time (Fig. 2C), which is particularly important when stimulation induces some movement (e.g., with electrical stimulation). Furthermore, the increased signal (compared with the thinner $z$ slices available with two-photon imaging) required lower laser strengths and offered faster acquisition. Using this technique, we were able to image at $>16 \mathrm{~Hz}$ for over an hour without visible photo damage. These relative benefits of 
a
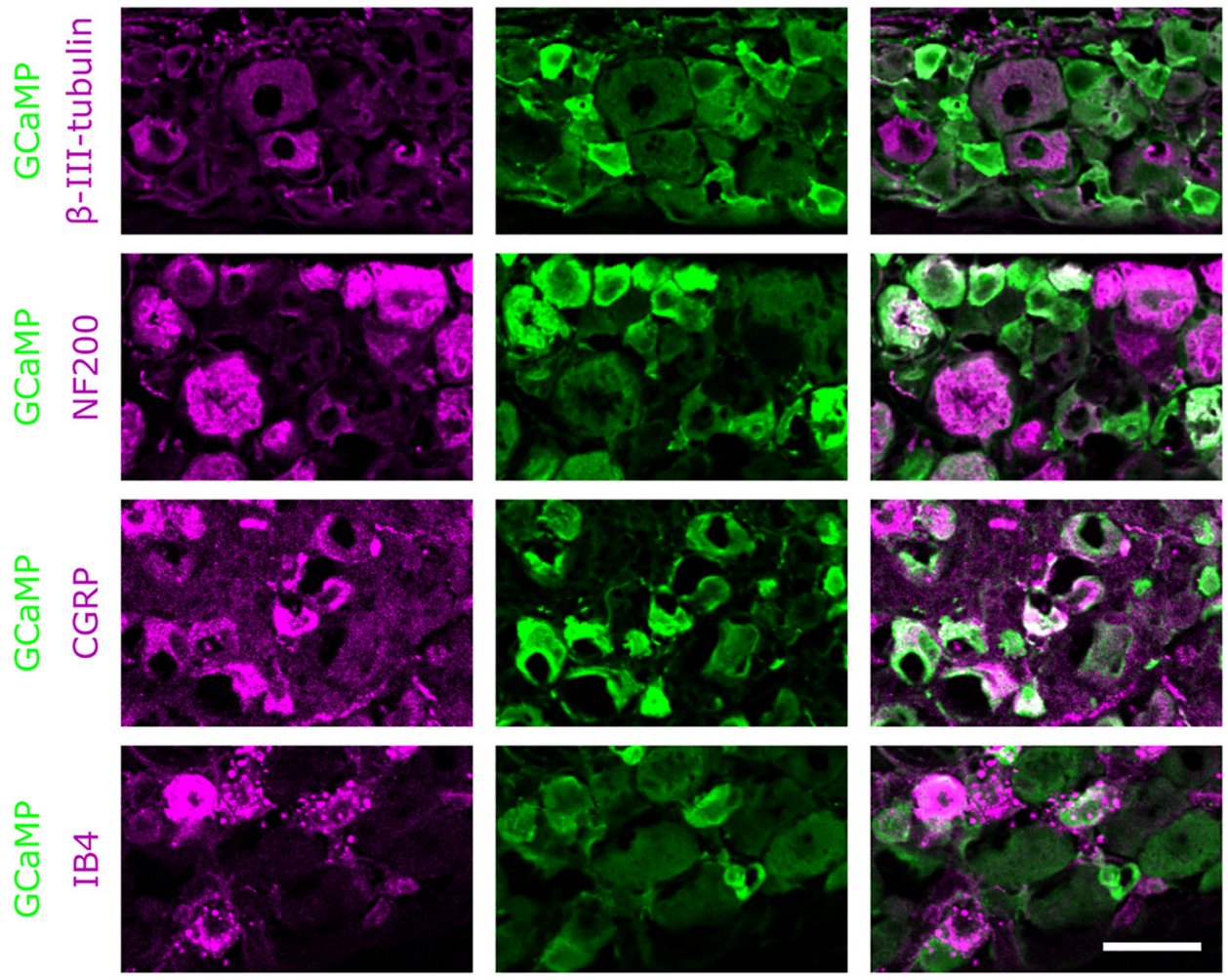

b

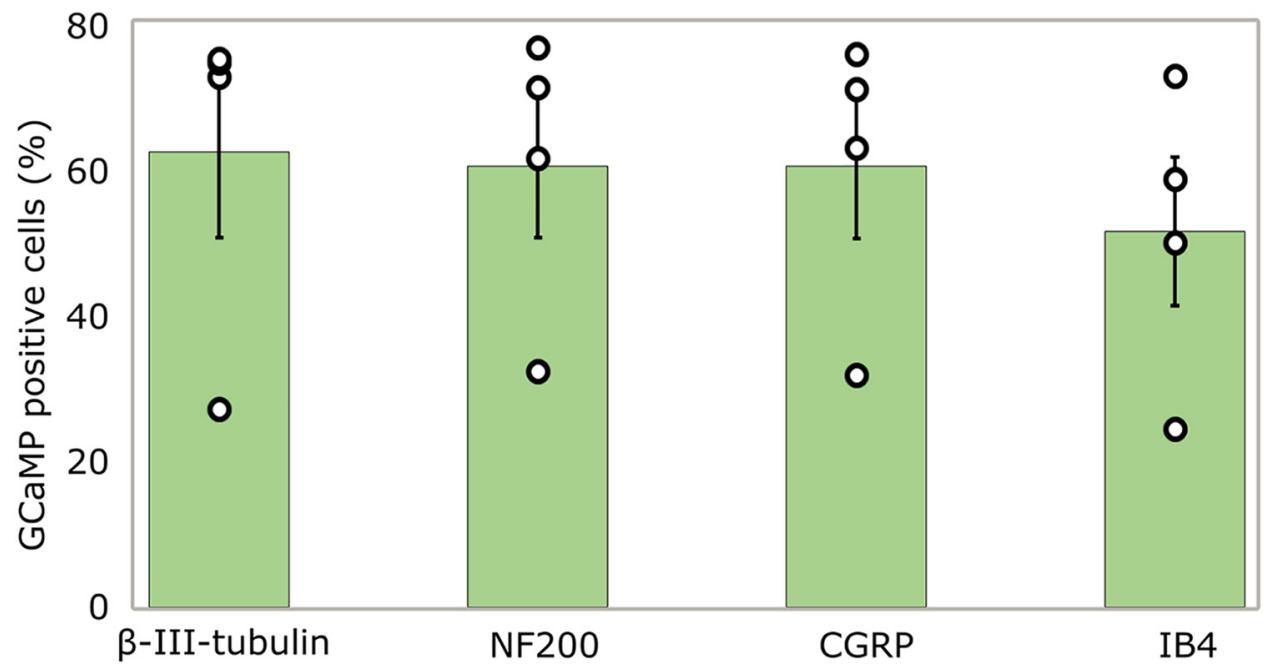

Figure 1. Intrathecal injections label a representative sample of DRG neurons. $\boldsymbol{A}$, Representative image of GCaMP-positive cells (green) as a proportion of all $\beta$-III-tubulin, NF200, CGRP, and IB4 immunoreactive neurons (magenta) after intrathecal injection. Scale bar: $50 \mu \mathrm{m}$. $\beta$-III-tubulin is a pan neuronal marker; NF200 labels large myelinated neurons; CGRP labels small peptidergic neurons; IB4 labels small, non-peptidergic fibers. B, Quantification of $\boldsymbol{A}$; the percentage of $\beta$-III-tubulin, NF200, CGRP, and IB4 immunoreactive cells that are also positive for GCaMP; $n=4$ mice. Green bar graphs represent mean \pm SEM while individual data points are displayed as empty circles.

confocal microscopy in this preparation may be especially pronounced due to the absence of a coverslip and the curved shape of the DRG, which result in a less regular air/sample interface and more out of focus tissue.

Using the approach outlined above it was possible to assess our minimal and maximal imaging range through comparison of cellular fluorescence at baseline (without any stimulation) and following cellular calcium accumulation $>30$ min after death (Fig. 2D). This revealed a large dynamic range over which we could visualize changes in intracellular calcium.

Using a $10 \times$ objective lens, we were typically able to image over 200 neurons simultaneously per DRG. Estimation of cell size shows an expected unimodal distribution with a majority of small diameter neurons and a tail of fewer, large diameter somas (Fig. 2E).

In the absence of stimulation most neurons had stable fluorescence with a SD of around $7 \%$. Only 79 of 1616 cell 
a

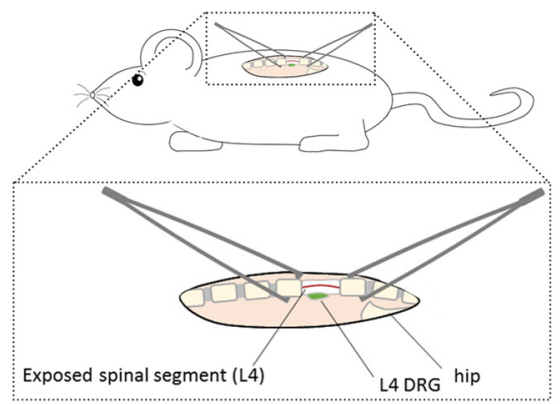

d

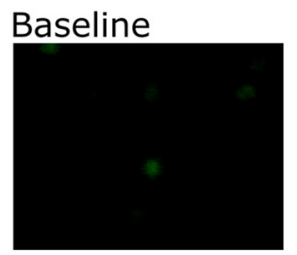

Post mortem

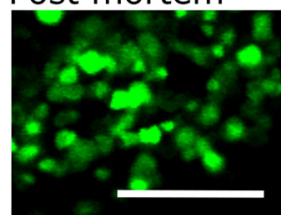

e

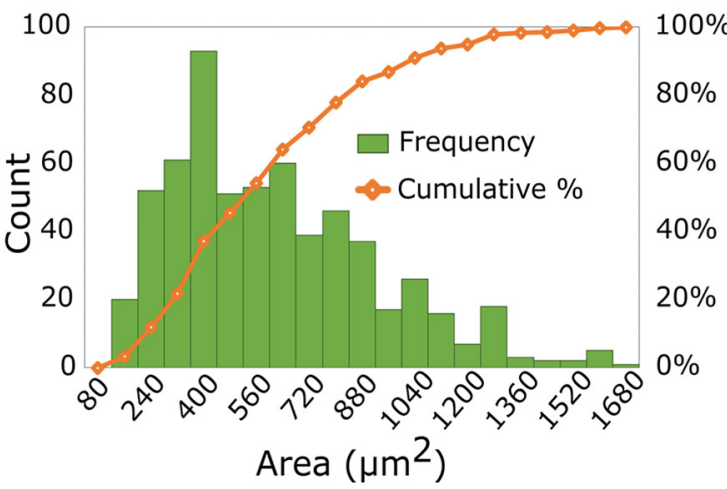

b
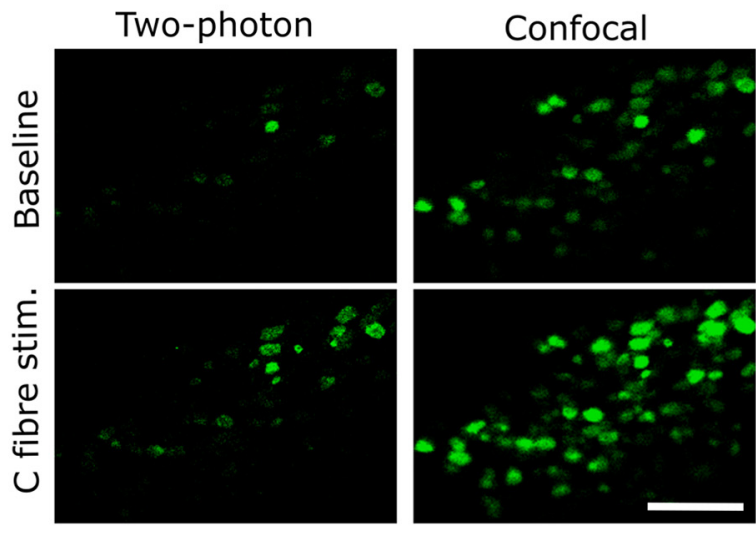

C
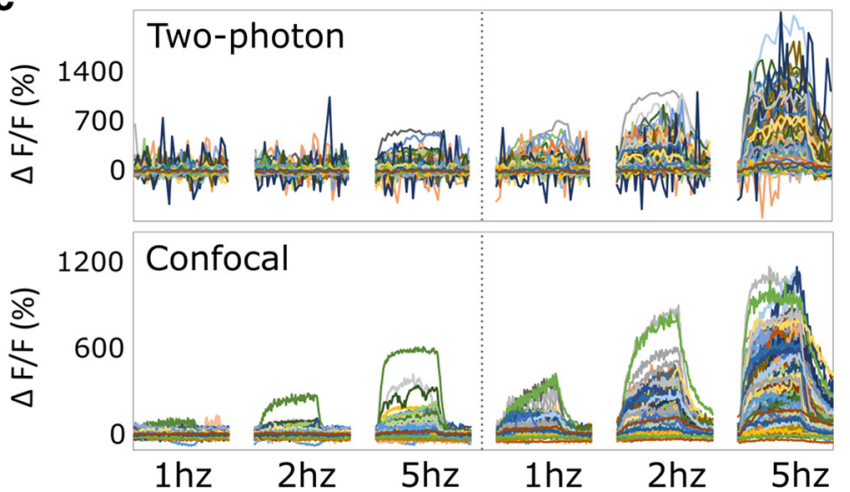

A fibre stim.

C fibre stim. $\overline{30 \mathrm{sec}}$

Figure 2. GCaMP labeled DRG neurons can be visualized in vivo using standard confocal microscopy. $\boldsymbol{A}$, Diagram showing the imaging set-up. The L4 DRG is exposed in a deeply anesthetized mouse, and the spinal column on either side of the exposed DRG is stabilized for in vivo confocal/two-photon imaging using spinal clamps attached to a custom-made stage. $\boldsymbol{B}$, Sample images of the DRG in vivo acquired under confocal (open pinhole) or two-photon mode. Confocal microscopy with an open pinhole reveals cellular GCaMP signal, which is lost in two-photon acquisition mode. Due to the more restricted slice thickness of multiphoton microscopy (left panel), fewer neurons are visible in the same field of view compared to when confocal microscopy is used with an open pinhole (right panel). This is particularly obvious during stimulation of the sciatic nerve (lower panel). Scale bar: $200 \mu \mathrm{m}$. $\boldsymbol{C}$, Fluorescence traces of $\boldsymbol{B}$. The sciatic nerve was stimulated at 1,2 , and $5 \mathrm{~Hz}$ at both $\mathrm{A}$ - and $\mathrm{C}$-fiber strengths to achieve a representative view of different response amplitudes both during confocal and multiphoton acquisition. In this preparation, a cleaner signal is generated when confocal microscopy with an open pinhole is used compared to two-photon microscopy. $\boldsymbol{D}$, Sample images of the DRG at baseline and following neuronal calcium accumulation $>30 \mathrm{~min}$ after death. GCaMP6s provides a large dynamic range over which to detect signal changes in vivo. Very little signal is evident when the animal is unstimulated (baseline) while a large increase in signal strength is evident following intracellular calcium accumulation $>30$ min after death (postmortem). Scale bar: $200 \mu \mathrm{m}$. $\boldsymbol{E}$, Frequency histogram and cumulative sum percentage of differentially sized cell bodies shows a skewed distribution with a larger percentage of smaller somata; $n=3456$ neurons in $n=13$ mice.

bodies $(\sim 5 \%)$ showed significant spontaneous fluctuations, which are likely to represent ongoing activity (from comparisons with evoked responses).

\section{Assessing the sensitivity of GCaMP6s sensory neuron imaging with electrical stimulation of the sciatic nerve}

Because of the novelty of our technique it was necessary to establish the relationship between action potential firing and calcium transients in DRG neurons. We took advantage of the fact that action potentials can be elicited in DRG neurons with a precisely controlled frequency and temporal pattern via electrical stimulation of peripheral nerves.
We used direct electrical stimulation of the sciatic nerve (which contains axons of the large majority of L4 DRG cell bodies), to induce activity in either large myelinated sensory neurons (A-fibers) or large and small afferents (A- and $\mathrm{C}$-fibers) at different frequencies. These stimulations can induce an action potential that can propagate into the DRG and depolarize the cell body.

We found that, with A-fiber strength stimulation, a subset of electrically responsive L4 DRG neurons showed increased fluorescence (Fig. $3 A-C$ ). The average magnitude of the A-fiber maximal response at $20 \mathrm{~Hz}$ was $654 \pm$ $974 \%$ of basal fluorescence. Supramaximal sciatic nerve stimulation activated the same neurons, but additionally recruited the large majority of remaining labeled neurons 


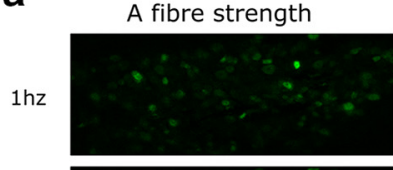

$2 \mathrm{hz}$

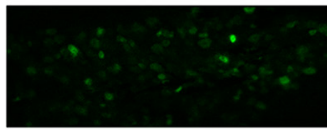

5 hz

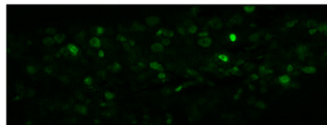

20 hz

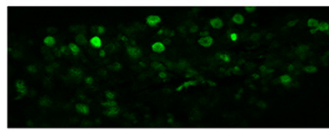

C fibre strength
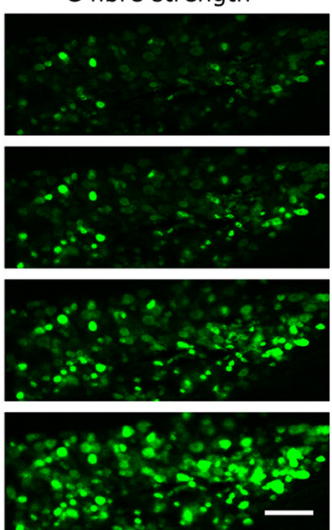

b

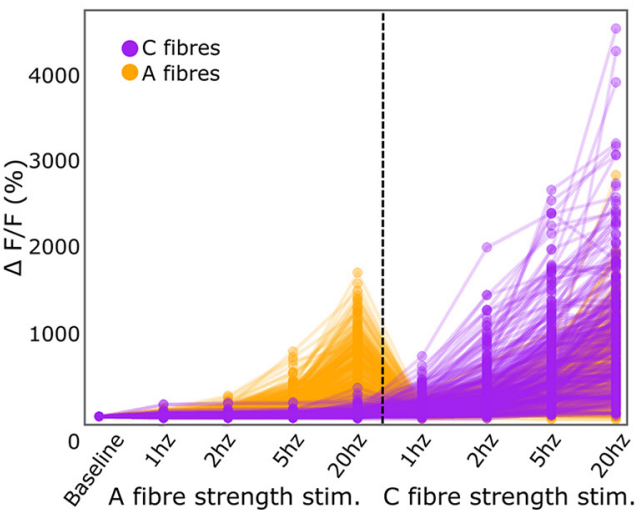

C

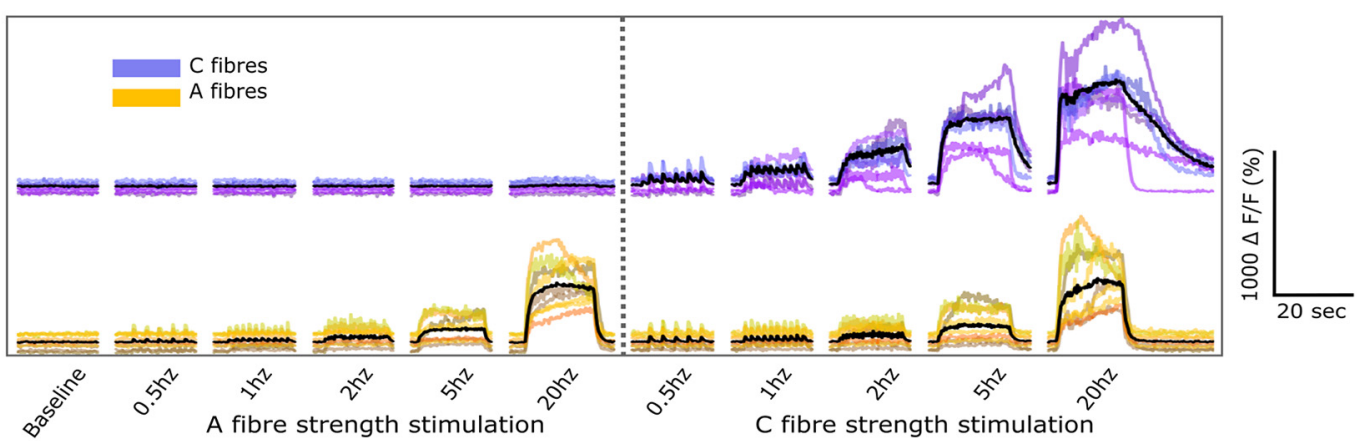

d

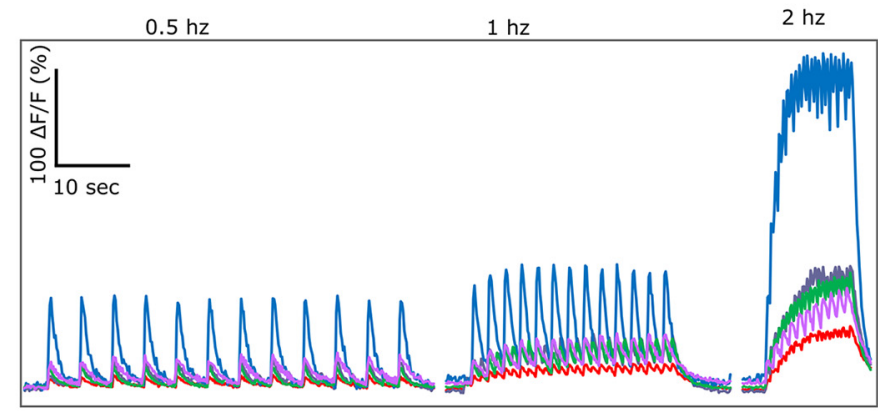

e

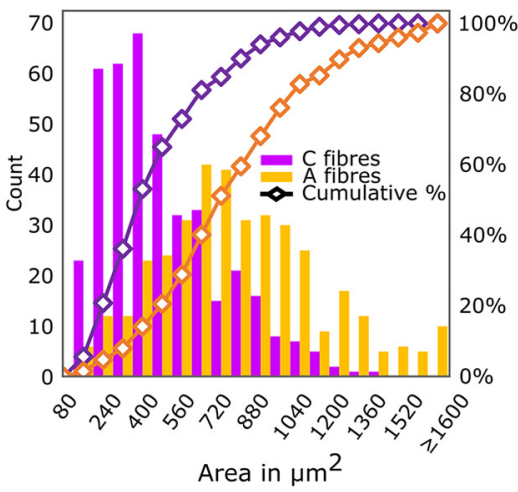

Figure 3. Electrical stimulation of the sciatic nerve leads to an increase in calcium signals in the DRG in an intensity and frequency dependent manner. A, Images of DRG cell bodies during direct stimulation of the sciatic nerve, at different frequencies and intensities. Scale bar: $200 \mu \mathrm{m}$. $\boldsymbol{B}$. Traces of individual cell bodies responding to electrical stimulation. Yellow lines represent neurons that respond to both A- and C-fiber strength stimulation and purple lines represent neurons that respond to C-fiber strength stimuli only. Data points displayed at averaged intensity over the period of stimulation; $n=774$ neurons in $n=6$ mice. $C$. Representative traces of GCaMP fluorescence during electrical stimulation. Electrical stimulation at A- and C-fiber strength resulted in activation of distinct subsets of neurons in a frequency dependent manner. Black lines represent averaged data. $\boldsymbol{D}$, Representative traces of GCaMP fluorescence during stimulation at C-fiber strength at low frequencies $(0.5,1$, and $2 \mathrm{~Hz})$ and high frame acquisition rate $(8 \mathrm{~Hz})$. Single action potentials generated by single electrical pulses to the sciatic nerve could be detected in the DRG. Each trace represents fluorescence from a single cell. E. Size distribution of cell bodies that respond to A- and C-fiber strength stimulation (yellow) compared to cell bodies that respond only to C-fiber strength stimuli (purple) and the cumulative sum of their sizes. Neurons responsive to A- and C-fiber strength stimulation are significantly larger compared to neurons which only respond to C-fiber strength stimuli: two-sample Kolmogorov-Smirnov test, $n=776$ neurons in $n=6$ mice, $p<0.001$. See also Video 1 for a recording of neuronal responses to electrical stimulation of the sciatic nerve.

in the L4 DRG. These additional cell bodies, which only responded to high intensity stimulation, were presumed to be neurons with C-fiber axons. They showed an average magnitude of $868 \pm 765 \%$ of basal fluorescence at their maximal response during $20-\mathrm{Hz}$ stimulation. In cell bodies of both $\mathrm{A}$ - and $\mathrm{C}$-fibers, the fluorescence intensity increased with stimulus frequency (Fig. $3 B, C$ ), suggesting that GCaMP6 fluorescence intensity provides a useful proxy 

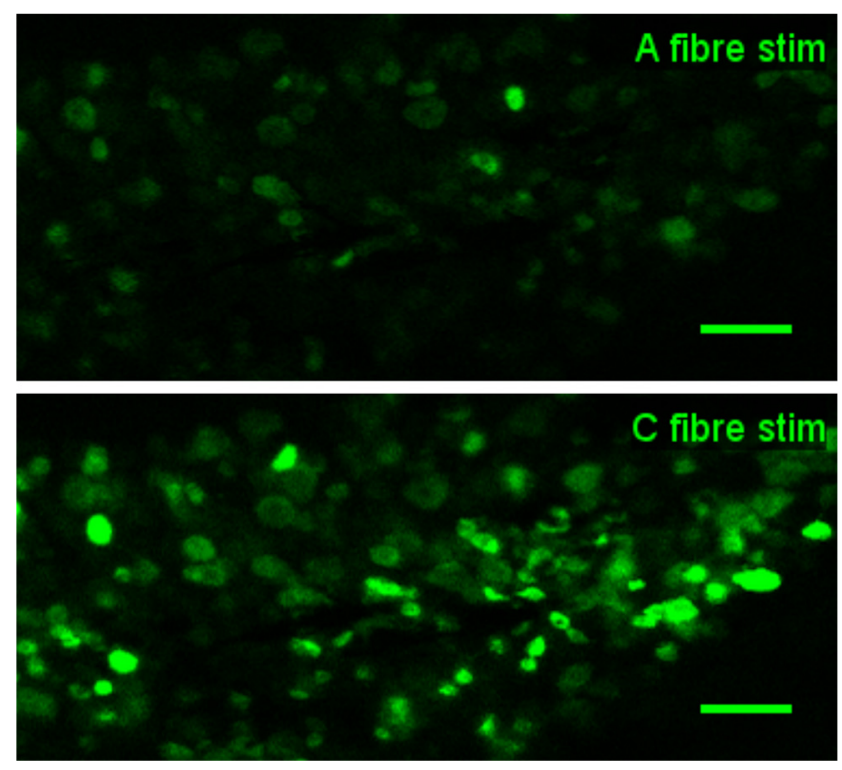

Video 1. Electrical stimulation of the sciatic nerve. L4 DRGs response to electrical stimulation of the ipsilateral sciatic nerve. GCaMP fluorescence was intensity and frequency dependent with recruitment of predominantly large diameter cell bodies during A-fiber strength stimulation and recruitment of additional, smaller diameter cell bodies with $\mathrm{C}$-fiber strength stimulation. Additionally, both during A- and C-fiber strength stimulation, the fluorescence intensity of neuronal cell bodies increased with stimulus frequency. Scale bar: $100 \mu \mathrm{m}$. See also Figure 3. [View online]

for frequency of action potential firing. See also Video 1 for a recording of neuronal responses to electrical stimulation of the sciatic nerve.

When the stimulation frequency was sufficiently low and the image acquisition rate high, a fluorescence signal was seen in response to the first of a train of stimuli as well as subsequent arriving impulse in the majority of neurons, revealing sufficient sensitivity to detect single action potentials (Fig. $3 C, D$ ). To ensure that only action potentials generated through stimulation of the sciatic nerve were recorded, the stimulated nerve was transected distally to the stimulation site. This procedure ensured an absence of contaminating spontaneous peripheral inputs, confirmed by the lack of response to paw pinching. The magnitude of this unitary response varied greatly, in some cases exceeding $100 \%$ of baseline fluorescence, and typically had a rise time of around $200 \mathrm{~ms}$ and an exponential decay with a time constant of $\sim 700 \mathrm{~ms}$.

As expected, A-fiber strength stimulation mostly activated larger DRG cell bodies while higher, C-fiber strength stimulation recruited new, smaller cells (Fig. 3E).

Out of a sample of 48 neurons detectably responding to A-fiber strength stimulation at $5 \mathrm{~Hz}$ (summated signal), 40 neurons also showed a detectable signal at $1 \mathrm{~Hz}$. Interestingly, in a sample of 62 cells detectably responding to C-fiber strength stimulation at $5 \mathrm{~Hz}$, all were also seen to respond at $1 \mathrm{~Hz}$ frequency. Thus, in the large majority of cases, we are able to detect the fluorescence change associated with a single action potential. However, it should be noted that individual action potentials were here detected by eye and statistical cutoff values to detect single action potentials are likely to be less precise. This is due to the relatively small change in fluorescence intensity generated by non-summating action potentials.

\section{Assessing polymodality in primary afferents}

To assess the level of polymodality within a neuronal population, all thermally responsive neurons were assessed for their responses to the opposing thermal modality (e.g., neurons observed responding to heat were similarly assessed for their response to cold), as well as their mechanical sensitivity.

A small proportion of heat-responding neurons also responded to decreases in temperature $(\sim 4 \%$ in 444 heatresponsive neurons, across six animals), while a larger proportion of cold responding neurons ( $\sim 40 \%$ in 31 coldresponsive neurons, across six animals) also encoded an increase in temperature (Fig. $4 A, B$ ). However, due to the very small number of cold-responsive neurons, these percentages varied greatly between experiments. Around 55\% of these temperature-responsive neurons were also responsive to pinch (Fig. $4 A-C$ ), revealing a subset of multimodaltemperature-sensitive neurons. See also Video 2 for a representative recording of polymodal responses in the DRG.

Size distribution showed that thermosensitive DRG somata were significantly smaller than cell bodies of the same cohort of animals that responded to stimulation by brush and A-fiber strength electrical stimulation (Fig. 4D). Indeed, cold-sensitive neurons represented the smallest pool of cell bodies, while neurons that were pinch-sensitive or responding to C-fiber strength stimulation were of average size, in agreement with the non-discriminative nature of these stimuli.

To ensure that our labeling technique did not lead to a selection bias, the responsiveness of temperature sensitive neurons to mechanical stimulation (pinch) was also assessed in two different lines of GCaMP6 transgenic mice (Fig. 4C). In all three approaches used (i.e., intrathecal injections of AAV9 carrying the GCaMP6 transgene, vs two types of transgenic animals expressing GCaMP6 constitutively in all neurons or conditionally only in peripheral neurons), polymodality was maintained above $48 \%$ (Fig. 4C).

\section{Assessing the effect of an intraplantar injection of formalin on prolonged DRG activity}

The formalin test is very widely used in pain research, but the mechanisms underpinning the responses are not completely understood. To address this question, we injected $20-\mu l$ formalin (1.85\% in saline) and vehicle into the plantar surface of the hind paw, while simultaneously imaging the ipsilateral DRG.

An injection of saline produced, as expected, a brief burst of activity, presumably from the pressure and/or damage associated with the injection. In contrast, the formalin injection induced a significantly greater response, much of it with a delayed activation (Fig. 5), with some neurons showing brief and sometimes repeated bursts of activity a few minutes after formalin injection (Fig. 5A). Indeed, the composite activity of DRG neurons mirrored pain behavior up to the second peak (Fig. 5). However, following the interphase, the average firing dropped considerably. Bouts of activity were still presented, mainly in small neurons, continuing 
a
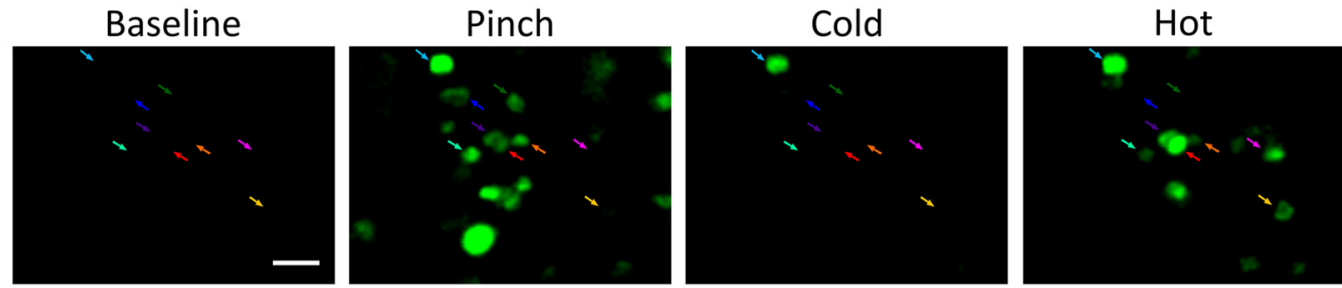

b

C
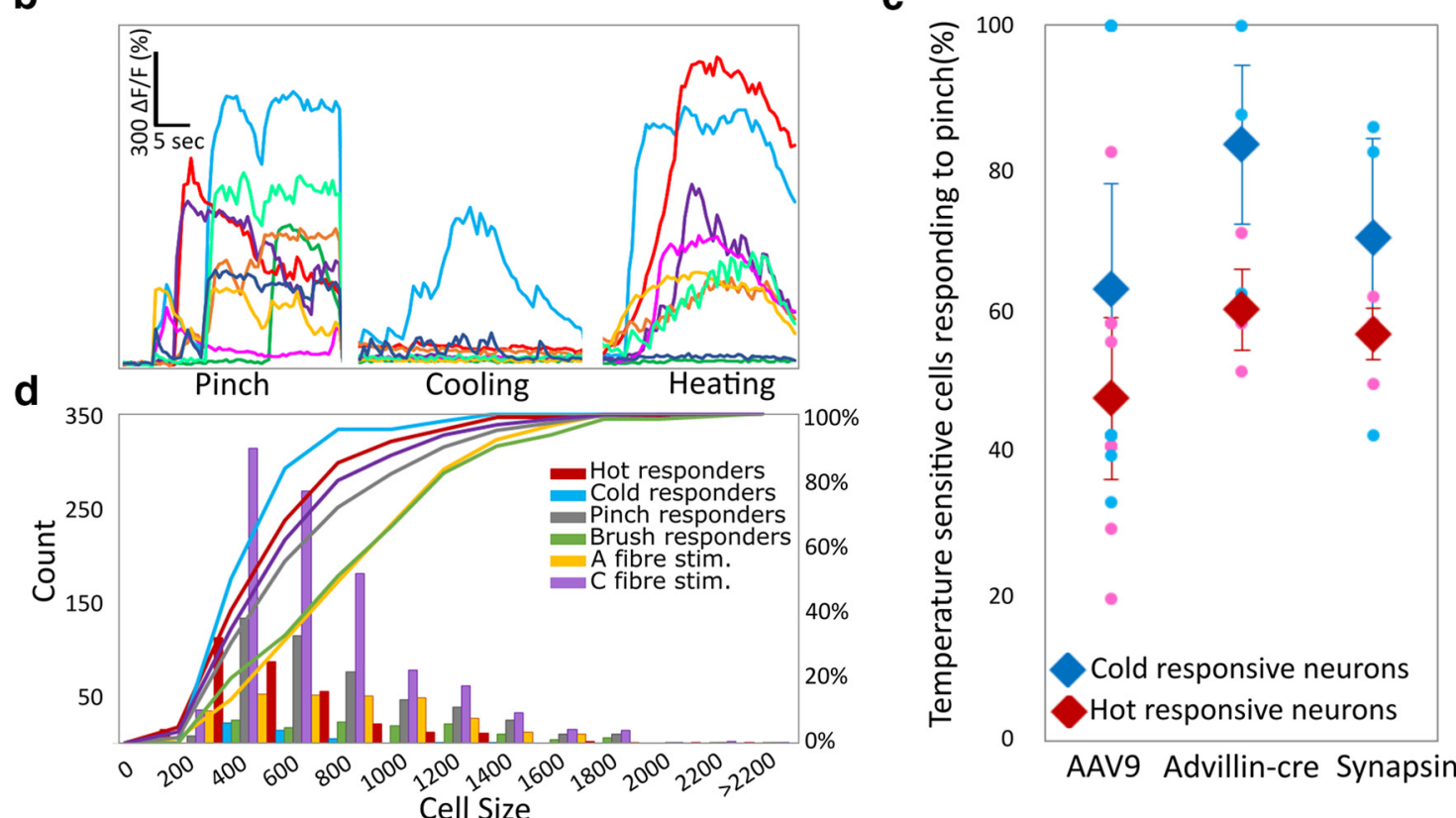

Figure 4. Polymodality is common in primary afferents. $\boldsymbol{A}$, Example images of DRG neurons responding to mechanical and thermal stimulation of the ipsilateral plantar paw. Scale bar: $50 \mu \mathrm{m}$. $\boldsymbol{B}$, Color coded example traces of neurons highlighted in $\boldsymbol{A}$. $\boldsymbol{C}$, Percentage of thermally sensitive DRG neurons responding also to noxious mechanical stimulation. Similar percentages of polymodal (thermally and mechanically sensitive neurons) were detected when using AAV9 to deliver GCaMP through intrathecal injections ( $n=6$ ) or when expressing GCaMP transgenically only in DRG neurons through GCaMP floxed mice expressing cre under the Advillin promoter (peripheral neurons; $n=3$ ) or when expressing GCaMP in all neurons through the Snap25 promoter $(n=3)$. Total $n=1138$ neurons. Blue data points show the percentage of cold sensitive neurons responding to pinch while red data points indicate the percentage of hot responding neurons also responding to pinch. Diamonds display the mean \pm SEM, circles indicate single mice. $\boldsymbol{D}$, Cell size distribution of cell bodies that respond to changes in temperatures versus cell bodies that respond to A-fiber strength stimulation, $\mathrm{C}$-fiber strength stimulation, brush and pinch, and the cumulative sum of their sizes. Cell bodies responsive to A-fiber strength stimulation, C-fiber strength stimulation, brush and pinch are significantly larger compared to somata which respond to changes in temperature: two-sample Kolmogorov-Smirnov test, total $n=1166$ neurons in $n=4$ mice, $p<0.001$ for all comparisons against temperature sensitive cell bodies, except temperature-responsive neurons versus C-fiber stimulation where $p=0.019$. See also Video 2 for a representative recording of polymodal responses in the DRG.

through the remainder of the observation period. Some have suggested that formalin induces a "wave" of activation as it spreads spatially in the tissue. We did not observe a sequential activation of different cell bodies. Rather individual neurons could stop and start, apparently randomly throughout this latter phase. Because this activity was of a low level and asynchronous among different neurons, the average rate of firing at these later times was not statistically different from saline treated animals.

\section{UVB irradiation leads to an increased sensitivity of thermally responsive neurons}

UVB irradiation is used as a model of inflammatory pain in humans and animals. We assessed the consequences of this treatment on the responsiveness of sensory neu- rons. We found that UVB inflammation consistently led to a striking increase in the responsiveness of temperature sensitive neurons (Fig. 6A,B). Indeed, warming of the injured paw led to significant increases in the responses of affected primary afferents which now revealed a linear increase in response with increases in temperature. This is compared with much lower levels of activation after heating of the uninjured paw. Here, low levels of heating had very limited effects on neuronal activity and much higher levels of stimulation were needed to achieve significant increases in neuronal responses (Fig. $6 A, B$ ). The sensitization of the peripheral nervous system was not limited to heating but also included enhanced sensitivity toward cooling of the injured paw. Even small decreases in temperature (e.g., to $27^{\circ} \mathrm{C}$ ) resulted in much greater 


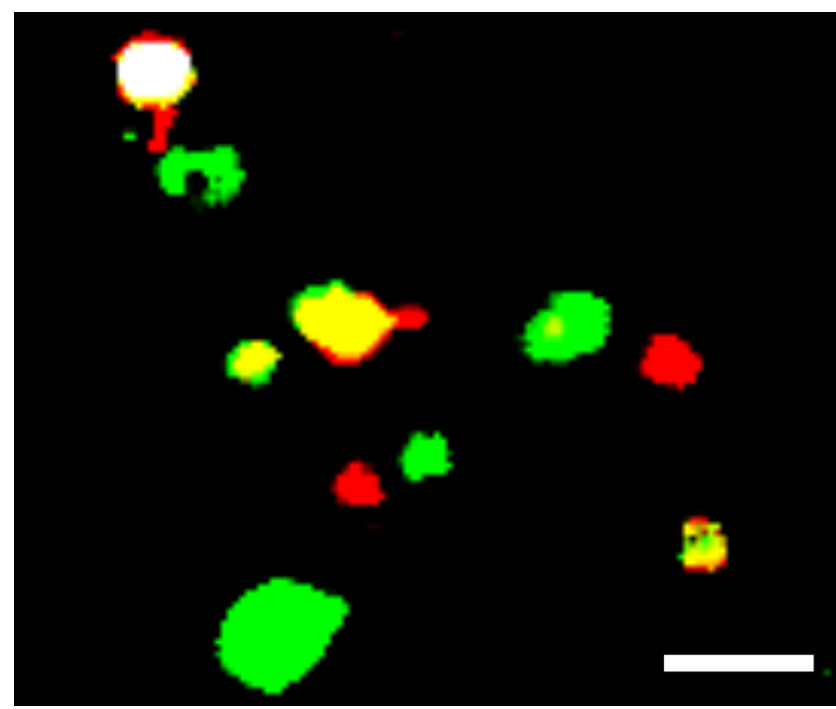

Video 2. Polymodality in DRG neurons. L4 DRGs response to peripheral cooling, heating, and noxious pinching of the ipsilateral paw. Neurons responding to pinching of the ipsilateral paw are green, neurons responding to heating are red and neurons responding to cooling are blue. A high level of polymodality is observed. Scale bar: $50 \mu \mathrm{m}$. For quantification, see also Figure 4. [View online]

activation of responding neurons during sterile inflammation as compared with responses in healthy control animals (Fig. $6 B)$. In addition, responses to mechanical stimuli, both noxious (pinch) and innocuous (brush), were significantly enhanced after UVB irradiation (Fig. 6C).

Interestingly, UVB irradiation did not lead to a significant increase in the percentage of neurons responding to thermal or mechanical stimulation of the irradiated paw, when compared to control anesthetized mice (Fig. 6D,E).

\section{Discussion}

Here, we use a new technique for the visualization and study of hundreds of peripheral neurons simultaneously in vivo. Using advanced genetically encoded calcium indicators, together with standard confocal microscopy, we were able to assess population responses of DRG neurons to electrical, thermal, mechanical and chemical stimuli, providing new insights into the processing of sensation and nociception in the peripheral nervous system during health and injury. While there have been a few reports using similar approaches, this is the first to report on large neuronal populations (typically $>200$ cell bodies in a single preparation), acquired at a subsecond frame rate.

The exposure and stabilization of the DRG involved a laterally extended laminectomy. Due to the encasing bony structure of the vertebrae, sufficient stabilization only required two vertebral clamps on either side of the exposure. However, the orientation of the DRG around the spinal cord meant that aligning the entire structure into one focal plane was challenging. As a result, confocal microscopy with an open pinhole was considered beneficial over two-photon microscopy in this instance. This approach is complementary to other methods, using both multi- and single-photon microscopes, aimed at increasing the acquisition of information in space while maintaining temporal resolution. Such methods include for example the use of a piezoelectric device (Callamaras and Parker, 1999), Bessel beams (Thériault et al., 2014), acoustooptical deflectors (Reddy and Saggau, 2005), multi-spot excitation (Bewersdorf et al., 1998; Kurtz et al., 2006), as well as various adaptations of scanning patterns (Göbel et al., 2007; Lillis et al., 2008). While our approach loses some of the added benefits often associated with twophoton microscopy, including greater penetration depth and reduced photo damage, it requires no modification of the microscope or software and provides a large dynamic range over which to detect changes in calcium signal, with a favorable signal-to-noise ratio and reduced effects of movement artefacts. In addition, the signal remained stable across long imaging sessions ( $>2 \mathrm{~h}$ ) with no detected bleaching.

The functionality of our system was validated by electrical stimulation of the sciatic nerve, activating DRG neurons in a predictable and reproducible manner (Fig. 3). The results of our experiments indicate that GCaMP6 fluorescence in DRG neurons is a sensitive reflection of neuronal activity, with good temporal resolution. Interestingly, our approach resulted in much greater numbers of neurons detected at much faster rates, compared to previously published works (Emery et al., 2016; Kim et al., 2016; Smith-Edwards et al., 2016). As expected, the majority of L4 DRG neurons responded to electrical stimulation in a frequency-dependent manner: low-threshold electrical stimuli recruited fewer neurons in the DRG, and these tended to have larger DRG somata. In contrast, high-threshold stimulation activated both the same large neurons and additionally many more (typically small diameter) neurons. Moreover, the GCaMP6 reporter was sensitive enough to detect calcium transients in the DRG elicited by single action potentials generated in the periphery.

At stimulation frequencies above $1 \mathrm{~Hz}$, temporal summation of the calcium transient meant that the fluorescence intensity also served as a proxy for the rate of action potential firing. The magnitude of calcium transients for a given level of activity varied between neurons. Some of this presumably represents variation in expression levels of GCaMP6s in different cell bodies, but it may also represent differences in calcium handling by different cell types (Scroggs and Fox, 1992; Malmberg and Yaksh, 1994; Westenbroek et al., 1998; Simons et al., 2009; Gemes et al., 2010).

Having established the sensitivity and specificity of the technique, we next investigated several aspects of the neurobiology underpinning sensory perception. Due to the novelty of the method, and several technical challenges it poses, it is essential to carefully examine novel claims that contrast traditionally held views. Indeed, one such claim suggests that polymodality, traditionally seen using microneurography, electrophysiology, multiple lines of in vitro evidence, and now also in vivo GCaMP imaging, was due to inflammation introduced through the use of invasive techniques (Emery et al., 2016). However, our data show that this is unlikely to be an adequate expla- 
a
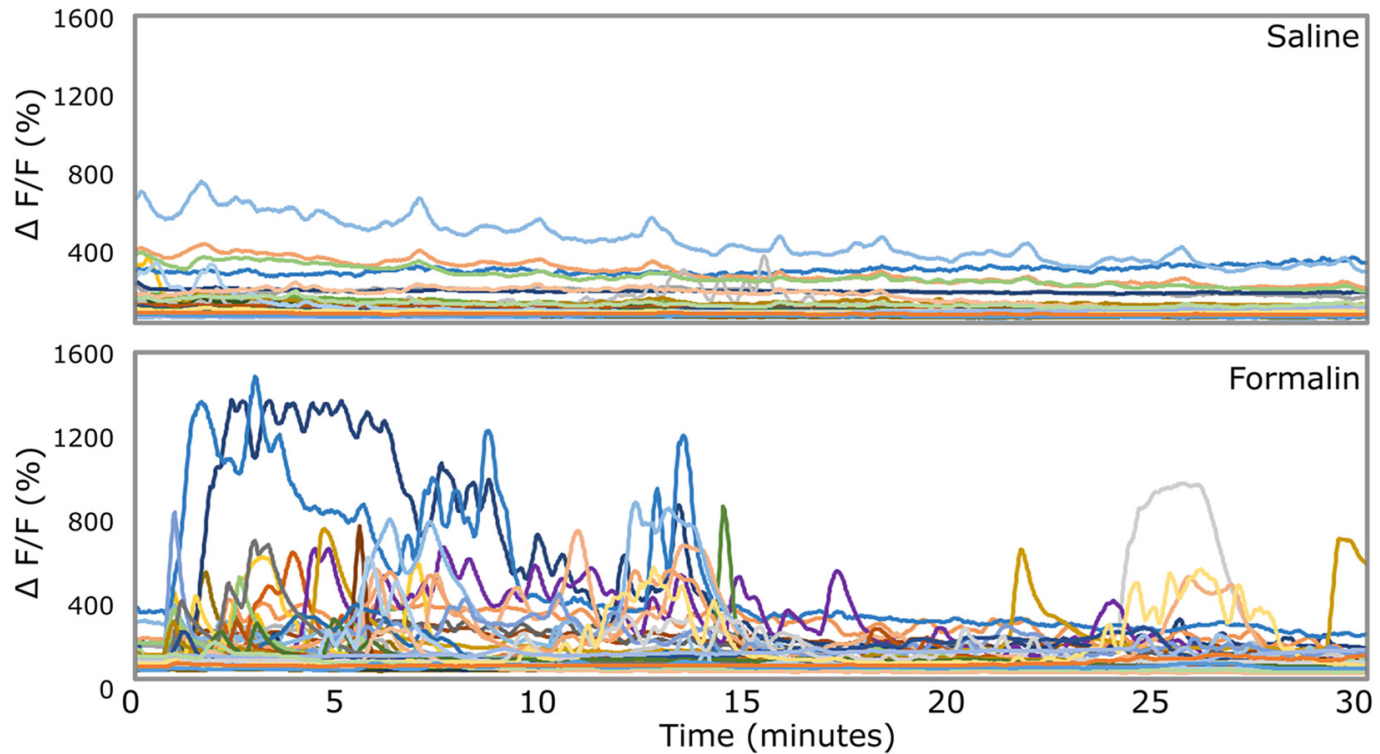

b

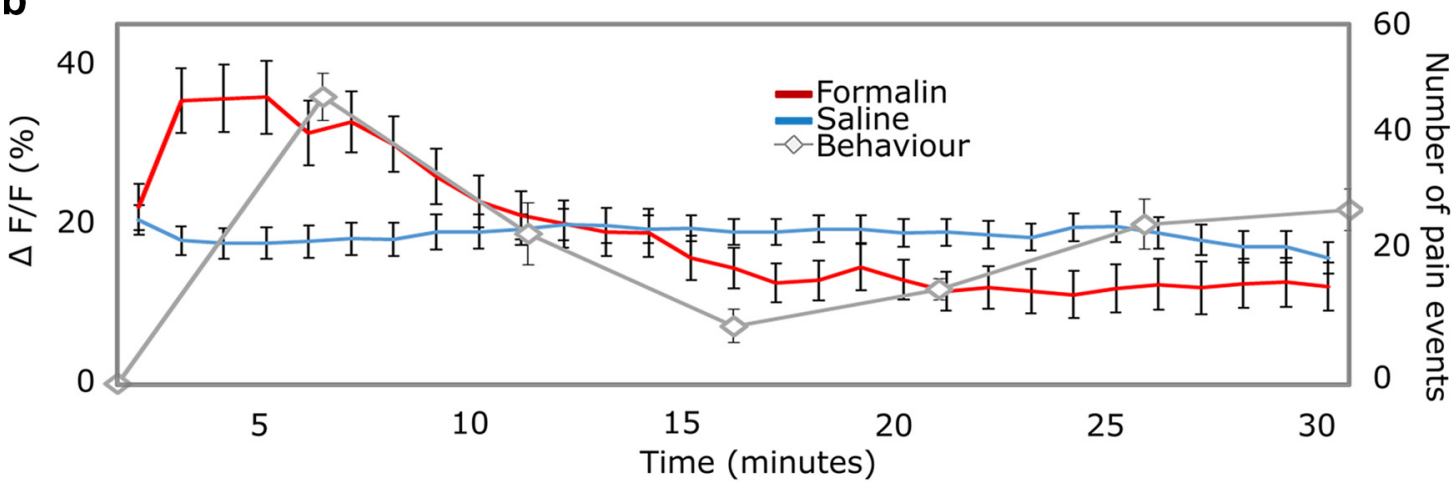

Figure 5. DRGs play a defining role in the generation of pain behavior in the formalin test. $\boldsymbol{A}$, Example traces of GCaMP fluorescence 0-30 min after intraplantar injection of saline (0.9\%) and formalin $(1.85 \%)$ in the same neurons. $\boldsymbol{B}$, Average response of DRG neurons ( $n=666$ neurons in $n=5$ mice, all subjected to saline and formalin) and behavioral response in mice $(n=8)$ after injection of saline and formalin into the plantar surface of the hind paw. Cellular response was averaged over 1 min and across neurons. Pain behavior was assessed as the number of pain events displayed by the mouse in 5-min increments. All data displayed as mean \pm SEM. Repeated-measures ANOVA, interaction of treatment (formalin vs saline) with time: $F_{(28,18,620)}=16.607, p<0.001$.

nation as the use of three different labeling techniques (including transgenic expression of GCaMP similar to that used by Emery et al., 2016) all resulted in similar levels of polymodality. It is possible that since Emery and colleagues imaged a smaller number of cell bodies, their results might have been skewed in unexpected ways, missing one of the most essential benefits of peripheral imaging, which is the collection of large sets of unbiased observations. A further reason for the discrepancy between their and our findings could be related to the statistical definitions of polymodality. The application of mechanically noxious stimuli also includes the application of non-noxious touch, necessarily activating a number of non-nociceptive neurons. By calculating the percentage of polymodal neurons using mechanical stimulation as the denominatory (i.e., percentage of thermally responsive neurons per mechanically responsive cell), Emery et al., are very likely to have significantly underestimated the percentage of polymodal neurons. In this manuscript, mechanical sensitivity is the numerator, meaning we calculated the percentage of mechanically responsive neurons per thermally responsive cell. This avoids diluting our results with touch sensitive neurons. We are perhaps still unavoidably underestimating the percentage of polymodal neurons by stimulating slightly different receptive fields with different stimulation modalities. We can therefore only comment on the minimum number of polymodal neurons, which is still significantly greater than that reported by Emery et al. (2016).

The visualization of large numbers of cell bodies with good temporal resolution can provide a reliable system with which to study the involvement of the peripheral nervous system in several acute and chronic pain conditions. The formalin test is one of the most widely used models for the study of pain, particularly in the pharmaceutical industry. Controversy still exists as to its mechanism of action, particularly relating to the relative role of the peripheral versus CNS. It has variably been suggested 
a
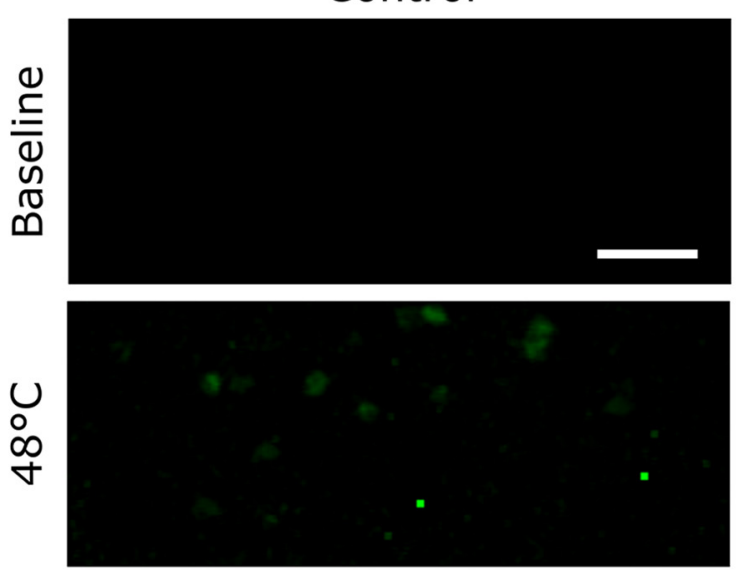

b

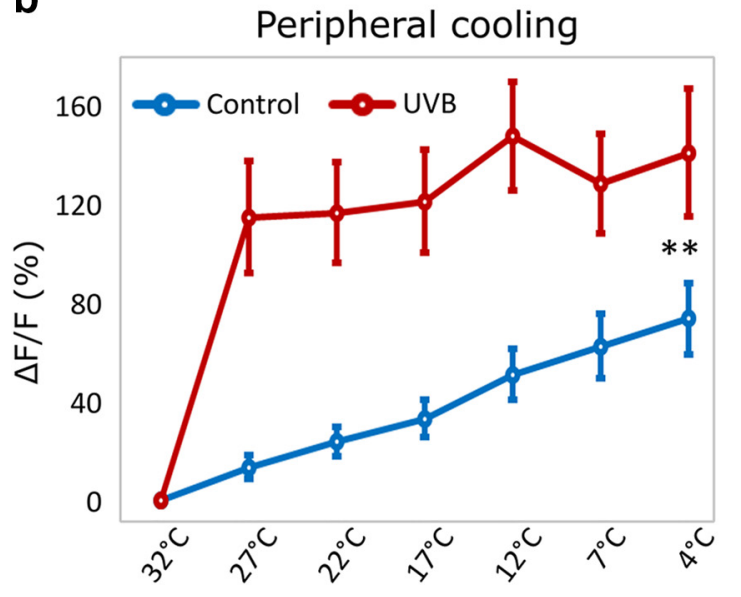

C

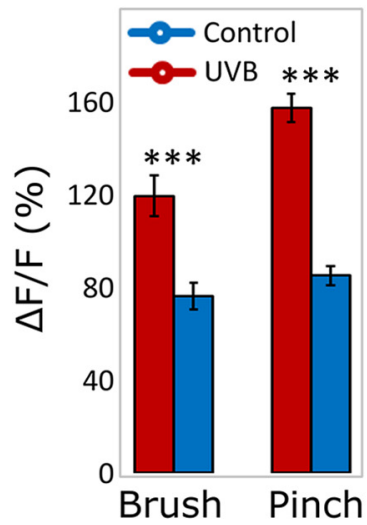

d

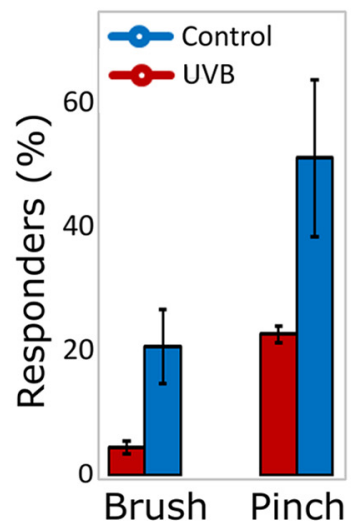

UVB
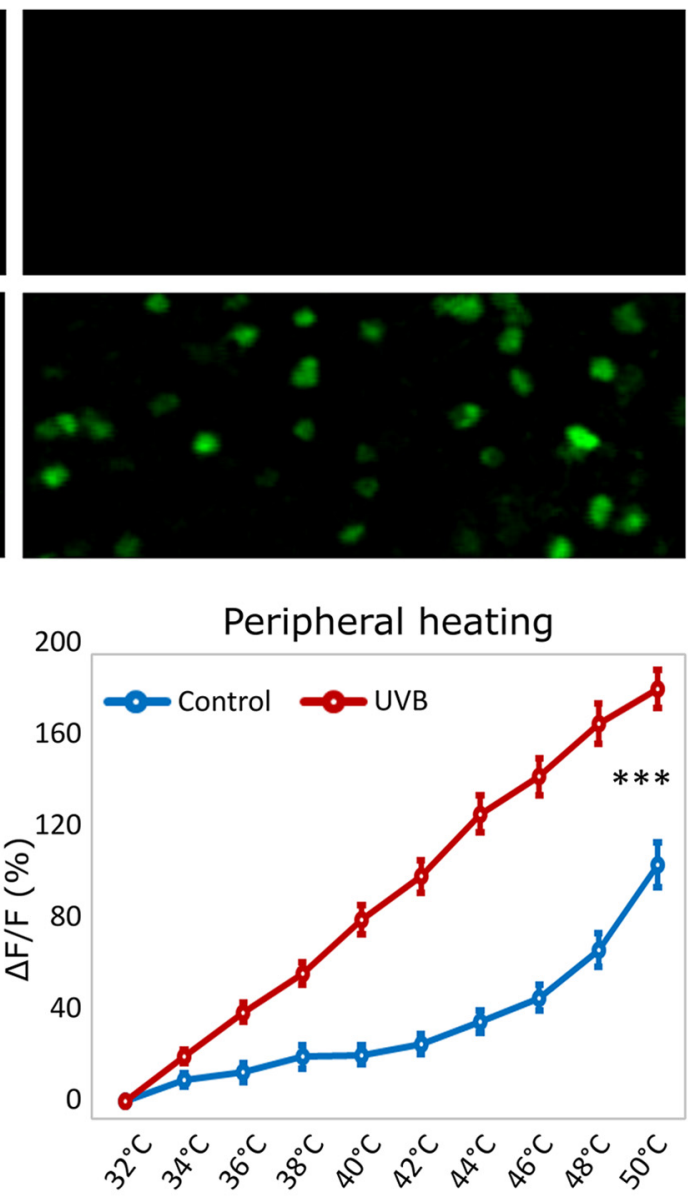

e

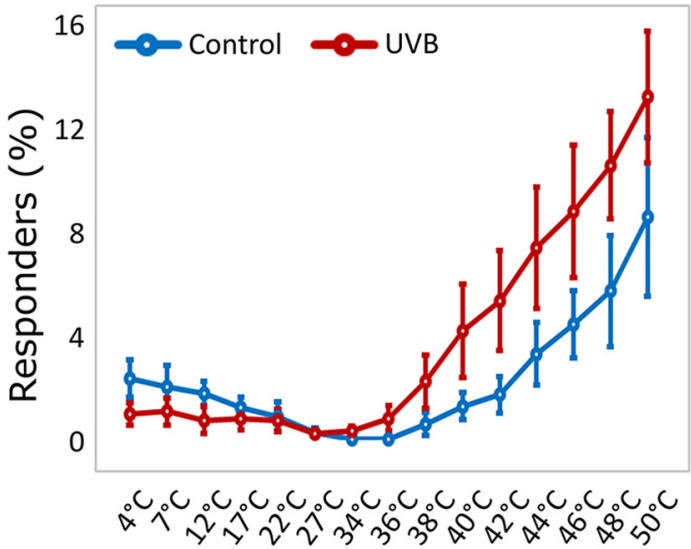

Figure 6. UVB irradiation increases the responsiveness of peripheral neurons. $\boldsymbol{A}$, Representative images of DRG neurons in mice stimulated with $32^{\circ} \mathrm{C}$ (baseline) and $48^{\circ} \mathrm{C}$ on the ipsilateral plantar surface, $48 \mathrm{~h}$ after UVB irradiation or control anesthesia. Scale bar: $100 \mu \mathrm{m}$. B . The response intensity of neurons stimulated thermally $48 \mathrm{~h}$ after UVB irradiation or control anesthesia. The response of neurons to both warming ( $n=393$ neurons) and cooling $(n=71$ neurons) of the ipsilateral paw is significantly greater in UVB-irradiated animals compared to controls. Differences were compared between groups in a split-plot ANOVA: For warming $F_{(1391)}=28.573$, $p<0.001$ and for cooling $F_{(1,69)}=11.421, p=0.001$. $C$, Response intensities of neurons stimulated mechanically (brush: $n=222$ neurons; pinch: $n=787$ neurons) $48 \mathrm{~h}$ after UVB irradiation are significantly greater as compared to neurons in sham irradiated mice (independent sample $t$ test, equal variances not assumed; for brush $t_{(149.742)}=4.112, p<0.001$. For pinch $t_{(730.186)}=9.848, p<$ 0.001). $\boldsymbol{D}$, The percentage of neurons responding to mechanical stimulation was not significantly different in UVB-irradiated animals compared to controls (independent sample $t$ test, equal variances not assumed; $t_{(2.112)}=2.696, p=0.108$ for neurons responding to brush and $t_{(2.0456)}=2.216, p=0.154$ for neurons responding to pinch). $\boldsymbol{E}$, The percentage of neurons responding to both warming and cooling of the ipsilateral paw was not significantly different in UVB-irradiated animals compared to controls (between groups difference 


\section{continued}

in a split-plot ANOVA, $\left.F_{(1,5)}=1.6, p=0.262\right)$. $* * * p<0.001$, $* * p<0.002$. For all experiments data displayed as mean $\pm \mathrm{SEM}, n=7$ mice (three control, four UVB).

that the second phase is generated directly by afferent activity (McCall et al., 1996) or by sensitization of the spinal dorsal horns (Coderre et al., 1990, 1993; Malmberg and Yaksh, 1992), or even supraspinal structures (Vaccarino and Melzack, 1989). The ability to visualize hundreds of DRG neurons simultaneously was well suited to investigate the relative role of the peripheral network in the generation of the formalin response.

Our results show high levels of DRG activation soon after an injection of formalin with a peak activity 5 min postinjection (Fig. 5). The cellular response was enhanced and delayed relative to saline, in line with previous work (Puig and Sorkin, 1996). We also found that neurons responded very heterogeneously. A small number fired tonically for long periods of time postinjection, and were likely responding to distention and/or damage associated with the injection (since similar responses were seen after saline injections). Beyond these, there were many neurons that were activated selectively by formalin. Some were activated soon after the injection, while others showed a more delayed response, either in the form of a single "burst" or of multiple cycles of activity across the observed period. In contrast to previous electrophysiological data (McCall et al., 1996; Henry et al., 1999), our recorded neurons did not seem, in aggregate, to constitute two clear phases of activity. Rather we saw strong activation of many neurons corresponding to the first phase of behavior, followed by low levels of intermittent firing seen in small numbers of predominantly very small neurons. We interpret this as evidence that the initial activation of neurons following a formalin injection might be the sensory drive for the first phase response. This activity also sensitizes dorsal horn neurons so that low levels of asynchronous activity in the periphery is able to generate a second phase in pain behavior, which is thought to be sustained by central sensitization and responsive to the application of pharmacological blockers in the spinal cord (for review, see McMahon et al., 1993). The low level of peripheral activity seen after a saline injection will impinge on a non-sensitized spinal cord, and hence does not initiate a long lasting behavioral change. In support of this view, early electrophysiological studies reported only low levels of C-fiber activity during the second phase of the formalin test (McCall et al., 1996). Such low levels are likely to be difficult to see clearly with calcium imaging but may provide sufficient input to activate a sensitized central pain pathway.

One interesting and highly translatable model of persistent pain is UVB-induced sterile inflammation. UVB irradiation leads to consistent swelling and hyperalgesia $48 \mathrm{~h}$ after injury. Here we were able to directly assess the involvement of the peripheral nervous system in the resulting hyperalgesia (Fig. 6). Indeed, we were able to visualize significant increases in sensitivity to peripheral heating and peripheral mechanical stimulation (both noxious and innocuous) as reported previously using electro- physiological methods (Bishop et al., 2010). Additionally, we also demonstrate for the first time, to the best of our knowledge, the involvement of the peripheral nervous system in UVB induced cold sensitivity. Indeed, we show that even small decreases in temperature had much more profound effects on peripheral responses after UVB irradiation as compared with responses from control animals. Interestingly, no significant differences were observed in the percentage of neurons responding to mechanical or thermal stimuli. This suggests limited unmasking of silent neurons with this model of sterile inflammation, and instead implicates mainly an increased activation of responding neurons.

It should be noted that in vivo imaging provides a significant advantage over traditional electrophysiological techniques in questions of cold processing. The limited number of cold sensitive primary afferents provides a practical barrier for the assessment of changes in peripheral cold processing using stochastic single cell approaches. Instead, the ability to sample hundreds of neurons simultaneously, as shown here, provides an excellent opportunity to investigate populations of rare neurons, including, but not limited to, cold-sensitive afferents.

In conclusion, we significantly enhanced the spatial and temporal sensitivity of a novel technique applied to the large-scale study of the peripheral nervous system. Using this technique, we were able to confirm the traditionally held, but recently challenged, view of polymodality in the peripheral nervous system. Additionally, we revealed new roles for peripheral processing in acute and persistent pain states.

\section{References}

Barretto RPJ, Gillis-Smith S, Chandrashekar J, Yarmolinsky DA, Schnitzer MJ, Ryba NJP, Zuker CS (2015) The neural representation of taste quality at the periphery. Nature 517:373-376. CrossRef

Bewersdorf J, Pick R, Hell SW (1998) Multifocal multiphoton microscopy. Opt Lett 23:655.

Bishop T, Marchand F, Young AR, Lewin GR, McMahon SB (2010) Ultraviolet-B-induced mechanical hyperalgesia: a role for peripheral sensitisation. Pain 150:141-152. CrossRef

Callamaras N, Parker I (1999) Construction of a confocal microscope for real-time $x-y$ and $x-z$ imaging. Cell Calcium 26:271-279. CrossRef

Chen Q, Cichon J, Wang W, Qiu L, Lee S-JR, Campbell NR, Destefino N, Goard MJ, Fu Z, Yasuda R, Looger LL, Arenkiel BR, Gan W-B, Feng G (2012) Imaging neural activity using Thy1GCaMP transgenic mice. Neuron 76:297-308. CrossRef Medline

Chen TW, Wardill TJ, Sun Y, Pulver SR, Renninger SL, Baohan A, Schreiter ER, Kerr RA, Orger MB, Jayaraman V, Looger LL, Svoboda K, Kim DS (2013) Ultrasensitive fluorescent proteins for imaging neuronal activity. Nature 499:295-300. CrossRef Medline

Coderre TJ, Katz J, Vaccarino AL, Melzack R (1993) Contribution of central neuroplasticity to pathological pain: review of clinical and experimental evidence. Pain 52:259-285. Medline

Coderre TJ, Vaccarino AL, Melzack R (1990) Central nervous system plasticity in the tonic pain response to subcutaneous formalin injection. Brain Res 535:155-158. 
Dana H, Chen TW, Hu A, Shields BC, Guo C, Looger LL, Kim DS, Svoboda K (2014) Thy1-GCaMP6 transgenic mice for neuronal population imaging in vivo. PLoS One 9:e108697. CrossRef Medline

Denk F, Ramer LM, Erskine EL, Nassar MA, Bogdanov Y, Signore M, Wood JN, McMahon SB, Ramer MS (2015) Tamoxifen induces cellular stress in the nervous system by inhibiting cholesterol synthesis. Acta Neuropathol Commun 3:74. CrossRef

Dombeck DA, Khabbaz AN, Collman F, Adelman TL, Tank DW (2007) Imaging large-scale neural activity with cellular resolution in awake, mobile mice. Neuron 56:43-57. CrossRef Medline

Emery EC, Luiz AP, Sikandar S, Magnúsdóttir R, Dong X, Wood JN (2016) In vivo characterization of distinct modality-specific subsets of somatosensory neurons using GCaMP. Sci Adv 2:e1600990. CrossRef

Flusberg BA, Nimmerjahn A, Cocker ED, Mukamel EA, Barretto RPJ, Ko TH, Burns LD, Jung JC, Schnitzer MJ (2008) High-speed, miniaturized fluorescence microscopy in freely moving mice. Nat Methods 5:935-938. CrossRef

Gemes G, Rigaud M, Koopmeiners AS, Poroli MJ, Zoga V, Hogan QH (2010) Calcium signaling in intact dorsal root ganglia. Anesthesiology 113:134-146. CrossRef

Ghosh KK, Burns LD, Cocker ED, Nimmerjahn A, Ziv Y, Gamal A, El, Schnitzer MJ (2011) Miniaturized integration of a fluorescence microscope. Nat Methods 8:871-878. CrossRef

Göbel W, Kampa BM, Helmchen F (2007) Imaging cellular network dynamics in three dimensions using fast 3D laser scanning. Nat Methods 4:73-79. CrossRef

Henry JL, Yashpal K, Pitcher GM, Coderre TJ (1999) Physiological evidence that the 'interphase' in the formalin test is due to active inhibition. Pain 82:57-63. Medline

Johannssen HC, Helmchen F (2010) In vivo Ca2 + imaging of dorsal horn neuronal populations in mouse spinal cord. J Physiol 588: 3397-3402. CrossRef Medline

Kandel ER, Schwartz JH, Jessell TM, Siegelbaum SA, Hudspeth AJ (2013) Principles of neural science, Ed 5. New York: McGraw-Hill.

Kim YS, Anderson M, Park K, Guan Y, Spray DC, Dong X, Shin Kim Y, Zheng Q, Agarwal A, Gong C, Young L, He S, Colleen LaVinka P, Zhou F, Bergles D, Hanani M (2016) Coupled activation of primary sensory neurons contributes to chronic pain. Neuron 91 : $1-12$.

Kurtz R, Fricke M, Kalb J, Tinnefeld P, Sauer M (2006) Application of multiline two-photon microscopy to functional in vivo imaging. J Neurosci Methods 151:276-286. CrossRef Medline

Lillis KP, Eng A, White JA, Mertz J (2008) Two-photon imaging of spatially extended neuronal network dynamics with high temporal resolution. J Neurosci Methods 172:178-184. CrossRef Medline

Malmberg AB, Yaksh TL (1992) Antinociceptive actions of spinal nonsteroidal anti-inflammatory agents on the formalin test in the rat. J Pharmacol Exp Ther 263:136-146.

Malmberg AB, Yaksh TL (1994) Voltage-sensitive calcium channels in spinal nociceptive processing: blockade of $\mathrm{N}$ - and P-type channels inhibits formalin-induced nociception. J Neurosci 14:48824890.

McCall WD, Tanner KD, Levine JD (1996) Formalin induces biphasic activity in C-fibers in the rat. Neurosci Lett 208:45-48. Medline

McMahon SB, Lewin GR, Wall PD (1993) Central hyperexcitability triggered by noxious inputs. Curr Opin Neurobiol 3:602-610.

McMahon SB, Koltzenburg M, Tracey I, Turk DC (2013) Wall and Melzack's textbook of pain. Philadelphia: Saunders.
Ottoni EB (2000) EthoLog 2.2: a tool for the transcription and timing of behavior observation sessions. Behav Res Methods Instrum Comput 32:446-449. Medline

Puig S, Sorkin LS (1996) Formalin-evoked activity in identified primary afferent fibers: systemic lidocaine suppresses phase- 2 activity. Pain 64:345-355. Medline

Reddy GD, Saggau P (2005) Fast three-dimensional laser scanning scheme using acousto-optic deflectors. J Biomed Opt 10:64038. CrossRef

Scroggs RS, Fox AP (1992) Multiple Ca* + currents elicited by action potential waveforms in acutely isolated adult rat dorsal root ganglion neurons. J Neurosci 12:1789-1801.

Sekiguchi KJ, Shekhtmeyster P, Merten K, Arena A, Cook D, Hoffman E, Ngo A, Nimmerjahn A (2016) Imaging large-scale cellular activity in spinal cord of freely behaving mice. Nat Commun 7:11450. CrossRef

Simons SB, Escobedo Y, Yasuda R, Dudek SM (2009) Regional differences in hippocampal calcium handling provide a cellular mechanism for limiting plasticity. Proc Natl Acad Sci USA 106: 14080-14084. CrossRef Medline

Smith-Edwards KM, DeBerry JJ, Saloman JL, Davis BM, Woodbury CJ (2016) Profound alteration in cutaneous primary afferent activity produced by inflammatory mediators. Elife 5 . CrossRef

Stosiek C, Garaschuk O, Holthoff K, Konnerth A (2003) In vivo two-photon calcium imaging of neuronal networks. Proc Natl Acad Sci USA 100:7319-7324. CrossRef Medline

Sun XR, Badura A, Pacheco DA, Lynch LA, Schneider ER, Taylor MP Hogue IB, Enquist LW, Murthy M, Wang SS-H (2013) Fast GCaMPs for improved tracking of neuronal activity. Nat Commun 4:2170. CrossRef Medline

Thériault $G$, Cottet $M$, Castonguay A, McCarthy $N$, De Koninck $Y$ (2014) Extended two-photon microscopy in live samples with Bessel beams: steadier focus, faster volume scans, and simpler stereoscopic imaging. Front Cell Neurosci 8:139.

Tian L, Hires SA, Mao T, Huber D, Chiappe ME, Chalasani SH, Petreanu L, Akerboom J, McKinney SA, Schreiter ER, Bargmann Cl, Jayaraman V, Svoboda K, Looger LL (2009) Imaging neural activity in worms, flies and mice with improved GCaMP calcium indicators. Nat Methods 6:875-881. CrossRef

Usoskin D, Furlan A, Islam S, Abdo H, Lönnerberg P, Lou D, HjerlingLeffler J, Haeggström J, Kharchenko O, Kharchenko PV, Linnarsson S, Ernfors P (2015) Unbiased classification of sensory neuron types by large-scale single-cell RNA sequencing. Nat Neurosci 18:145-153. CrossRef

Vaccarino AL, Melzack R (1989) Analgesia produced by injection of lidocaine into the anterior cingulum bundle of the rat. Pain 39:213219. Medline

Westenbroek RE, Hoskins L, Catterall WA (1998) Localization of $\mathrm{Ca} 2+$ channel subtypes on rat spinal motor neurons, interneurons, and nerve terminals. J Neurosci 18:6319-6330. Medline

Williams EK, Chang RB, Strochlic DE, Umans BD, Lowell BB, Liberles SD (2016) Sensory neurons that detect stretch and nutrients in the digestive system. Cell 166:209-221. CrossRef Medline

Wu A, Dvoryanchikov G, Pereira E, Chaudhari N, Roper SD (2015) Breadth of tuning in taste afferent neurons varies with stimulus strength. Nat Commun 6:8171. CrossRef

Zariwala HA, Borghuis BG, Hoogland TM, Madisen L, Tian L, De Zeeuw Cl, Zeng H, Looger LL, Svoboda K, Chen T-W (2012) A Cre-dependent GCaMP3 reporter mouse for neuronal imaging in vivo. J Neurosci 32:3131-3141. CrossRef Medline 